\title{
Knowledge about mammography screening in Germany by education and migrant status - results of a cross-sectional study (InEMa)
}

Eva-Maria Berens ${ }^{1 * \dagger}$, Simone Kaucher ${ }^{2 \dagger}$, Sandra van Eckert ${ }^{3}$, Maren Reder ${ }^{4,5}$, Petra Kolip ${ }^{4}$ and Jacob Spallek ${ }^{3}$

\begin{abstract}
Background: The population-based mammography screening program (MSP) is aimed to reduce breast cancer mortality, to detect breast cancer at an early stage, and to allow for less invasive treatment. However, it also has some potential harms, such as overdiagnosis and overtreatment. Therefore, it is necessary that women receive sufficient and balanced information to enable informed decision-making. We examined knowledge about benefits and harms of the MSP in Germany among first-time invitees of different socio-demographic backgrounds.

Methods: This observational study assessed knowledge about benefits and harms of the MSP among women who were invited to the MSP for the first time by six multiple choice items, using a postal survey. We investigated (i) single items of knowledge, (ii) the distribution of sufficient knowledge stratified by education, migration status and invitation, and (iii) possible determinants of sufficient knowledge by analyzing Odds Ratios (ORs) using bivariate and multivariate logistic regression.

Results: In total, 5397 women included in the analyses. 46.1\% of the study population had sufficient knowledge about benefits and harms of the MSP. However, women with low educational level and migration background had higher proportions of insufficient knowledge and used most frequently the option "don't know". Women had the most difficulties answering the numeric question and the question about the target group correctly. Results from the logistic regression showed that the odds of having sufficient knowledge were higher among well-educated women (OR 3.84, 95\% Cl 3.24-4.55), among women who already received the MSP invitation (OR 1.38, 95\%Cl 1.201.59) and lowest among Turkish women (OR 0.14, 95\%Cl 0.07-0.25).

Conclusions: Women with low education and migration background need adapted information regarding benefits and harms of the MSP and are important target groups for further developing the information material about mammography screening to reduce disparities in knowledge and enable informed decision-making.
\end{abstract}

Keywords: Mammography screening program, Knowledge, Education, Migrants, Benefits, Harms

\footnotetext{
* Correspondence: eva-maria.berens@uni-bielefeld.de

tEva-Maria Berens and Simone Kaucher contributed equally to this work.

${ }^{1}$ Department of Health Services Research and Nursing Science,

Interdisciplinary Center for Health Literacy Research, School of Public Health,

Bielefeld University, P.O. Box 1001 31, 33501 Bielefeld, Germany

Full list of author information is available at the end of the article
}

(c) The Author(s). 2019 Open Access This article is distributed under the terms of the Creative Commons Attribution 4.0 International License (http://creativecommons.org/licenses/by/4.0/), which permits unrestricted use, distribution, and reproduction in any medium, provided you give appropriate credit to the original author(s) and the source, provide a link to the Creative Commons license, and indicate if changes were made. The Creative Commons Public Domain Dedication waiver (http://creativecommons.org/publicdomain/zero/1.0/) applies to the data made available in this article, unless otherwise stated. 


\section{Background}

Breast cancer is the most prevalent cancer among women in Germany. In 2014, there were about 69,000 new cases of female breast cancer [1]. Thus, until 2009, the German government implemented a population-based mammography screening program (MSP) to reduce breast cancer mortality, detect breast cancer at an early stage, and allow for less invasive treatment $[2,3]$. Women aged 50 to 69 years are invited to receive mammography within a certified program every two years. However, the MSP also has some potential harms, such as overdiagnosis, overtreatment, false-positive and false-negative results [4-6]. As the harm-benefitconsiderations of the MSP are discussed critically, it is necessary that women receive sufficient and balanced information to enable them to make an individual informed choice [6], which is based on sufficient knowledge about benefits and harms of the procedure and the personal attitude. Women in Germany receive an information brochure providing information about the procedure, benefits and possible harms of the MSP along with the invitation to the screening [2]. However, previous results from Germany showed that only $27.1 \%$ of women who were invited to the MSP for the first time made an informed choice [7]. Overall, knowledge was poor (mean knowledge score 2.81 on a scale from 0 to 7), lower educated and women with a migration background had even lower mean knowledge, with mean knowledge scores of 2.23 and 2.20, respectively [7].

International studies also showed that knowledge about benefits and harms of the MSP is relatively poor [8-13]. Women tend to overestimate benefits and underestimate harms of the MSP and women thought that participation would protect them from developing breast cancer $[9,10$, 12]. Many studies have shown a positive association between educational level and knowledge about benefits and harms of mammography screening [10, 11, 13-15]. However, most studies assessed knowledge either in intervention contexts, e.g. testing information sources $[8,16]$, or assessed knowledge unrelated to a decision need [9-11]. Studies about knowledge among migrant women is scarce. There is also a lack of studies about knowledge closely related to the (first) decision. Assessing the knowledge of first time deciders may be especially useful because the decision will not be influenced by habit or previous experience, which is an important factor for their screening decision [17]. Furthermore, detailed analyses on single knowledge items and determinants of knowledge in Germany, especially among vulnerable groups, are scarce.

Therefore, this article will focus on knowledge at the time of the first decision for or against participation in the MSP and (i) describe different aspects of knowledge about benefits and harms of the MSP, (ii) describe the distribution of sufficient knowledge stratified by educational level, migration status and invitation, and (iii) analyse possible determinants of sufficient knowledge.

\section{Methods}

This article is based on data of the InEMa-study (Informierte Entscheidung zur Teilnahme am MammographieScreening-Programm-study, engl. Informed Choice for Participation in Mammography Screening Programstudy), which is an observational study investigating informed choice among women in Germany who were invited to the population-based MSP for the first time. Data were collected between October 2013 and July 2014 [18].

\section{Study population}

Women were invited to participate in this study one to two months before their 50th birthday, since women also receive the invitation to participate in the populationbased MSP at this time. The target populations' addresses were retrieved from local registry-offices within the area of Westphalia-Lippe, which is part of the federal state of North Rhine-Westphalia, Germany. Women with a Turkish migration background were identified using a namebased approach and received all study materials in German and Turkish. Women with a previous breast cancer diagnosis and who already had participated in the MSP were excluded from analyses, since they were not part of the target population for screening and did not make a decision for the first time.

\section{Variables}

Knowledge about benefits and harms of the MSP was the outcome of interest. It was assessed with five context and one numeric question which were created based on previous research [19, 20]. For more details see the study protocol [18] and previous analyses regarding the instrument [21, 22]. Topics covered were (1) screening for people without symptoms, (2 - numeric question) frequency of positive screening results, (3) false-positives, (4) false-negatives, (5) diagnoses with the MSP, and (6) overdiagnosis and overtreatment. All questions were multiple choice questions. A score of 1 indicated a correct answer, a score of 0 an incorrect answer. Missing responses and "don't know" responses were categorized as incorrect. Based on the validation analyses of the knowledge questionnaire which had shown that the discrimination parameters were different between items (Item 1: .256, Item 2: .403 , Item 3: .805, Item 4: .812, Item 5: .120, Item 6: .433), a summary index weighted by these discrimination parameters was calculated [22]. Accordingly, the knowledge scale ranged from 0 to 2.8 points. Since there were no agreed criteria for defining sufficient knowledge, the midpoint of the scale was set as the cut-off point according to previous research $[19,20,23]$. Thus, women reaching a score of lower than 1.4 were defined as having "insufficient knowledge" and women with a score of 1.4 to 2.8 were categorized as having "sufficient knowledge" as suggested previously [22]. 
Educational level was defined using school degree and years of school education. Women with no degree or at least 9 years of school education were categorized as having low education, 10 or 11 years of education was defined as medium education and women with at least 12 years of education were considered as having high education. Migration status was assessed by asking for country of origin and citizenship. Women were categorized into nonimmigrants, Turkish migrants, resettlers (ethnic German migrants from Eastern European countries with a resettler status) and migrants from other countries. Furthermore, the variable invitation defined whether women already received the invitation for the MSP.

Additionally, family history of breast cancer, advice on mammography by a gynaecologist, previous mammography experience, intention to perform mammography, attitude towards mammography and health insurance status were assessed.

\section{Statistical analyses}

Descriptive analysis was performed to characterize the study population and show the distribution of the single knowledge questions stratified by educational level, migration status, and invitation. Logistic regression was used to investigate factors associated with knowledge about benefits and harms of the MSP. First, bivariate logistic regression was performed and Odds Ratios (ORs) with 95\% confidence intervals $(95 \% \mathrm{CIs})$ were calculated to check for significant associations. In a second step, all significant variables were included in a multivariate model. All statistical analyses were performed using SPSS 25.

\section{Results}

In total, 17,349 women were invited to the study and 5839 women returned questionnaires and written consent forms (response rate 33.7\%). After excluding women who already had a breast cancer diagnosis $(n=179)$, women who had already participated in the MSP $(n=256)$, and women who had missing information on any of these variables $(n=15)$, we finally included 5397 individuals in the analyses.

Table 1 presents the distribution of educational level, migration status and invitation within the study population. The majority of the study population were German women $(n=4855,90 \%)$, had a medium educational level ( $n=2240,41.5 \%)$, and had received an invitation including an information brochure to the MSP before the study ( $n=4113,76.2 \%)$.

Overall, a mean knowledge-score of 1.27 (range 0-2.8) was found among the study population. Table 2 presents the single knowledge items among the study population. Only $15 \%$ of women knew that the MSP is aimed at healthy women, and $17.1 \%$ correctly assessed the frequency of positive screening results. $67.7 \%$ of women knew that there might be false-positive results, and $52.1 \%$
Table 1 Characterization of study participants $(n=5397)$

\begin{tabular}{lll}
\hline & $n$ & $\%$ \\
\hline Educational level & 1138 & 21.1 \\
Low & 2240 & 41.5 \\
Medium & 1952 & 36.2 \\
High & 67 & 1.2 \\
Missing & & \\
Migration status & 4855 & 90.0 \\
Non-immigrant & 223 & 4.1 \\
Resettler & 149 & 2.8 \\
Turkish & 164 & 3.0 \\
Others & 6 & $<0.1$ \\
Missing & & \\
Invitation & 4113 & 76.2 \\
Received & 1247 & 23.1 \\
Not received & 37 & 0.7 \\
Missing & & \\
\hline
\end{tabular}

knew about the possibility of false-negative results. While $18.9 \%$ correctly indicated that women performing mammography screening are more likely to get a diagnosis compared to women not performing the screening, $40 \%$ of women knew about the possibility of overdiagnosis and overtreatment.

The results stratified by educational level, migration status and invitation are presented in the Additional file 1. The mean knowledge score and the percentage of correct answers for each question increased with increasing educational level and with received invitation. Non-immigrant women almost always had higher percentages of correct answers, compared to immigrant women but there was great variation among different immigrant groups. The answer option "don't know" was always more frequently used among low-educated and immigrant women.

Table 3 shows the distribution of sufficient knowledge by various co-variates and presents crude and adjusted ORs with corresponding 95\%CIs and $p$-values. Overall, $46.1 \%(n=2488)$ of the study population showed sufficient knowledge regarding benefits and harms of the MSP. However, sufficient knowledge varied widely by different study characteristics: among all women with low educational level $24.6 \%$ had sufficient knowledge, among those with high educational level $58.3 \%$ had sufficient knowledge. Among Turkish migrants the proportion of sufficient knowledge was $8.2 \%$ whereas it was $29.1 \%$ among the resettler group. Women with a negative attitude towards screening had a higher proportion of sufficient knowledge than women with a postitive attitude $(69.9 \%$ vs. $44.5 \%)$. Educational level, migration status, invitation status, type of health insurance, intention to perform mammography and attitude towards 
Table 2 Response frequencies for each knowledge item

\begin{tabular}{|c|c|c|c|}
\hline \multicolumn{2}{|c|}{ Knowledge questions } & \multirow{2}{*}{$\begin{array}{l}\text { Answer options } \\
\text { one who is healthy }\end{array}$} & \multirow{2}{*}{$\frac{\mathrm{n}(\%)}{810(15.0 \%)}$} \\
\hline 1 & When does one participate in mammography screening? & & \\
\hline & & one who has a change or knot in the breast & $231(4.3 \%)$ \\
\hline & & in both cases & $3990(73.9 \%)$ \\
\hline & & don't know & $157(2.9 \%)$ \\
\hline & & missing & 209 (3.9\%) \\
\hline \multirow[t]{6}{*}{2} & \multirow{6}{*}{$\begin{array}{l}200 \text { women participate } 20 \text { years in the mammography } \\
\text { screening program: How many have a positive result } \\
\text { warranting further diagnostics? }\end{array}$} & $1-20$ of 200 & $1464(27.1 \%)$ \\
\hline & & $21-50$ of 200 & $1332(24.7 \%)$ \\
\hline & & $51-100$ of 200 & $921(17.1 \%)$ \\
\hline & & $101-200$ of 200 & $80(1.5 \%)$ \\
\hline & & don't know & $1528(28.3 \%)$ \\
\hline & & missing & $72(1.3 \%)$ \\
\hline \multirow[t]{4}{*}{3} & \multirow[t]{4}{*}{ Does a positive result mean that a woman has breast cancer? } & yes & $868(16.1 \%)$ \\
\hline & & no & $3653(67.7 \%)$ \\
\hline & & don't know & $816(15.1 \%)$ \\
\hline & & missing & $60(1.1 \%)$ \\
\hline \multirow[t]{4}{*}{4} & \multirow{4}{*}{$\begin{array}{l}\text { Does the mammography screening program discover every } \\
\text { breast cancer? }\end{array}$} & yes & $691(12.8 \%)$ \\
\hline & & no & $2811(52.1 \%)$ \\
\hline & & don't know & 1849 (34.3\%) \\
\hline & & missing & $46(0.9 \%)$ \\
\hline \multirow[t]{5}{*}{5} & \multirow[t]{5}{*}{ Who is more likely to get a breast cancer diagnosis? } & women participating in MSP & $1019(18.9 \%)$ \\
\hline & & women not participating in MSP & $1152(21.4 \%)$ \\
\hline & & both the same & $2348(43.5 \%)$ \\
\hline & & don't know & $801(14.8 \%)$ \\
\hline & & missing & $77(1.4 \%)$ \\
\hline \multirow[t]{4}{*}{6} & \multirow{4}{*}{$\begin{array}{l}\text { Are some women treated for breast cancer although the } \\
\text { breast cancer would never have caused problems in } \\
\text { their life? }\end{array}$} & yes & $2158(40.0 \%)$ \\
\hline & & no & $729(13.5 \%)$ \\
\hline & & don't know & $2428(45.0 \%)$ \\
\hline & & missing & $82(1.5 \%)$ \\
\hline
\end{tabular}

Correct answers are indicated in bold

mammography were significantly associated with sufficient knowledge in bivariate regressions, whereas a family history of breast cancer, gynaecological advice and previous mammography experience showed no significant associations with sufficient knowledge about the MSP. Women with a medium and high educational level had an about two to almost four times higher chance of having sufficient knowledge than women with a low educational level (OR 2.47, 95\%CI 2.10-2.91 and OR $3.84,95 \%$ CI 3.24-4.55) when adjusting for migration status, invitation, type of health insurance, intention to perform mammography and attitude towards mammography. Women with a migration background showed a lower chance of having sufficient knowledge than non-immigrant women. Women with a Turkish migration background had the lowest chance (OR 0.14, 95\%CI 0.07-0.25) for sufficient knowledge, followed by resettler women (OR 0.43, 95\%CI
$0.32-0.59$ ) and women coming from other countries (OR 0.45 , 95\% CI 0.31-0.65) irrespective of their educational background, invitation, type of health insurance, intention to participate and attitude towards mammography. Women who had already received an invitation to participate in the MSP including the information brochure had an almost $40 \%$ higher chance of having sufficient knowledge compared to women who had not received the MSP invitation yet (OR 1.38, 95\%CI 1.20-1.59). Furthermore, women with a private health insurance were more likely to have sufficient knowledge compared to women with a statutory health insurance (OR 1.32, 95\%CI 1.05-1.68). Women with a positive attitude towards the MSP showed lower odds of having sufficient knowledge, compared to women with a negative attitude towards the MSP (OR 0.46, 95\%CI 0.36$0.60)$. Women who had the intention to undergo a mammography within the MSP were less likely to have sufficient 
Table 3 Determinants of sufficient knowledge with bivariate and adjusted Odds Ratios

\begin{tabular}{|c|c|c|c|c|c|}
\hline \multicolumn{2}{|c|}{ Sufficient knowledge (knowledge score $\geq 1.4$ ) } & \multicolumn{2}{|l|}{ Bivariate models } & \multicolumn{2}{|l|}{ Adjusted model } \\
\hline & $n(\%)$ & OR $(95 \% \mathrm{Cl})$ & $p$-value & OR $(95 \% \mathrm{Cl})$ & $p$-value \\
\hline Total & $2488(46.1 \%)$ & & & & \\
\hline Educational level & $n=2469$ & & & & \\
\hline Low & $280(24.6 \%)$ & Ref. & $<.001$ & Ref. & $<.001$ \\
\hline Medium & $1052(47.0 \%)$ & $2.71(2.32-3.18)$ & & $2.47(2.10-2.91)$ & \\
\hline High & $1137(58.3 \%)$ & $4.28(3.64-5.03)$ & & $3.84(3.24-4.55)$ & \\
\hline Migration status & $n=2486$ & & & & \\
\hline Non-immigrant & $2362(48.7 \%)$ & Ref. & $<.001$ & Ref. & $<.001$ \\
\hline Resettler & $65(29.1 \%)$ & $0.43(0.32-0.58)$ & & $0.43(0.32-0.59)$ & \\
\hline Turkish & $12(8.2 \%)$ & $0.10(0.05-0.17)$ & & $0.14(0.07-0.25)$ & \\
\hline Others & $47(28.5 \%)$ & $0.42(0.30-0.59)$ & & $0.45(0.31-0.65)$ & \\
\hline Invitation & $n=2479$ & & & & \\
\hline Not received & $517(41.5 \%)$ & Ref. & $<.001$ & Ref. & $<.001$ \\
\hline Received & $1962(47.8 \%)$ & $1.29(1.14-1.47)$ & & $1.38(1.20-1.59)$ & \\
\hline Health insurance & $n=2468$ & & & & \\
\hline Statutory & 2249 (45.0\%) & Ref. & $<.001$ & Ref. & .017 \\
\hline Private & $219(61.7 \%)$ & $1.97(1.58-2.45)$ & & $1.32(1.05-1.68)$ & \\
\hline Attitude & $n=2486$ & & & & \\
\hline Negative & $239(69.9 \%)$ & Ref. & $<.001$ & Ref. & $<.001$ \\
\hline Positive & 2247 (44.5\%) & $0.35(0.27-0.44)$ & & $0.46(0.36-0.60)$ & \\
\hline Intention & $n=2486$ & & & & \\
\hline No & $479(55.4 \%)$ & Ref. & $<.001$ & Ref. & .015 \\
\hline Yes, opportunistic & $198(41.8 \%)$ & $0.58(0.46-0.73)$ & & $0.73(0.57-0.93)$ & \\
\hline Yes, MSP & 1649 (45.3\%) & $0.67(0.58-0.78)$ & & $0.77(0.65-0.91)$ & \\
\hline Yes, no idea where & $96(43.4 \%)$ & $0.62(0.46-0.83)$ & & $0.74(0.54-1.03)$ & \\
\hline No response & $64(33.2 \%)$ & $0.40(0.29-0.56)$ & & $0.63(0.44-0.91)$ & \\
\hline Family history of breast cancer & $n=2482$ & & & & \\
\hline No & $2158(46.0 \%)$ & Ref. & .583 & & \\
\hline Yes & $324(47.2 \%)$ & $1.05(0.89-1.23)$ & & & \\
\hline Gynaecological advice & $n=2486$ & & & & \\
\hline No & 1060 (45.2\%) & Ref. & .254 & & \\
\hline Yes & $1426(46.8 \%)$ & $1.07(0.96-1.19)$ & & & \\
\hline Mammography experience & $n=2481$ & & & & \\
\hline No & $888(45.8 \%)$ & Ref. & .635 & & \\
\hline Yes & $1593(46.5 \%)$ & $1.03(0.92-1.15)$ & & & \\
\hline
\end{tabular}

knowledge than women who did not intend to undergo mammography (OR 0.77, 95\%CI 0.65-0.91).

\section{Discussion}

\section{Main findings}

The aim of this paper was to determine knowledge about benefits and possible harmful effects of mammography screening among women eligible for the MSP for the first time. This is, to our knowledge, the first study presenting detailed results about knowledge on benefits and harms of the
MSP stratified by educational level and migration status in Germany. The results showed that overall, $46.1 \%$ of the participants had sufficient knowledge, however, there are large differences when sufficient knowledge was investigated separated by educational level and migration status. Only $24.6 \%$ of women with a low education level had sufficient knowledge. Women with migration background showed great disparities. Only $8.1 \%$ of women with a Turkish migration background and about 30\% of resettler women and women coming from other countries had sufficient knowledge about 
benefits and harms of the MSP. Further, type of health insurance, whether women had received the invitation for the MSP participation, intention to undergo mammography and attitude towards the MSP were significantly associated with sufficient knowledge.

\section{Comparison with other studies}

These results are in line with findings from previous research that found in general poor knowledge about benefits and harms of the MSP [8-13]. In contrast, a study from the Netherlands showed much better knowledge scores [15]. An explanation might be that they included more questions about organizational factors, such as compulsory participation which might have been easier to answer than questions about scientific core-concepts such as false-positive or false-negative results or a comparison of indicators compared to women not performing screening as included in this study. Furthermore, knowledge in the Dutch study was measured by yes/no answers rather than by multiple choice options. This needs to be considered when comparing our results to findings from other studies. But in general, as different studies use different definitions and cut-offs, it is difficult to compare the actual knowledge of women.

Previous studies have shown that women especially tend to overestimate benefits and to underestimate harms of screening programs $[8,10,11,13]$. In our study, women underestimated the number of diagnosis within the screening program compared to not performing screening. Further aspects were not numerically assessed. However, almost 70\% knew about the possibility of false-positive and about $50 \%$ knew about the possibility of false-negative results.

Our results confirm previous international research indicating that poor knowledge is particularly observed among women with a low educational level $[10,11,13-$ 15]. The lower chance of having sufficient knowledge about benefits and harms of the MSP among immigrant women might be explained by poorer German language skills. The extremely low odds for sufficient knowledge among Turkish women in this study might be explained by the language of data collection. Providing women with a Turkish questionnaire enabled all women to respond - including women with no or very limited German language skills. Contrastingly, the invitation and the enclosed information brochure of the MSP are only distributed in German (different language versions are available online but are not send by mail, i.e., accessing the Turkish version requires more effort than accessing the German version). Therefore, many Turkish women with rather poor German language skills were able to answer our questionnaire, but were not able to read and understand the information brochure which was sent with the invitation to the MSP.
Especially the numeric question, asking for the breast cancer incidence among MSP participants, showed differences by educational level and migration status. Women with a low educational level and immigrant women, particularly women with a Turkish migration background, responded that they didn't know the correct answer. Numeric questions have also previously been shown to be difficult to answer $[8,13,14]$. Therefore, we did not ask for the correct number but gave (very wide) numeric ranges as answer options. Nonetheless, numeric concepts seem to be difficult to answer for women.

Another explanation for poor knowledge about benefits and harms of the MSP might be low level of health literacy. Especially migrants and persons of low educational level have been shown to perceive more difficulties in processing health information [24]. However, we did not assess health literacy in this study.

We also observed higher odds of having sufficient knowledge when women had received an invitation to the MSP. Thus, the information included in the accompanying brochure seemed to increase women's knowledge about the procedure, benefits and harms of the MSP. However, it is also possible that women have higher motivation to gather information about benefits and harms after receiving the invitation to the MSP itself because they are aware of the procedure and prompted to make a choice. Our results showed a lower proportion of correct answers, compared to an evaluation study of the German information brochure [11]. However, this may be explained by a different study procedure. Whereas women in the trial received questionnaire and brochure together, knowledge in our study was measured after women received the brochure and some time to contemplate their decision and access additional information about the MSP. Since we didn't ask the study participants whether they had read and understood the information brochure, we cannot differentiate whether the positive impact of the invitation letter was due to the information brochure itself or due to the fact that women needed to make a decision.

\section{Strengths and limitations}

A strength of our study is that the questionnaire was sent at about the same time as the invitation letter and measured knowledge in a realistic and not a trial context. Marteau and colleagues [19] noted that knowledge should be measured before the choice of (non-)participation is made. Furthermore, it is known that habit is an important factor for screening behavior [17]. Thus, knowledge at the time of the first decision is especially important. In addition, we were able to identify Turkish women based on a name-based algorithm and to send them the questionnaire in German and Turkish. To our knowledge, this is the first study investigating knowledge 
about benefits and harms of the MSP separated by migration status.

One limitation of this study is that women with a low educational level and women with a migration background were underrepresented. Thus, knowledge in our study is likely to be overestimated. Furthermore, immigrant women with poor German language skills, except for women with a Turkish migration background, might not have been able to respond since the questionnaire was only distributed in German and Turkish language. This would also have led to an overestimation of knowledge among immigrant women. Furthermore, we decided to use the mid-point of the knowledge scale to define sufficient knowledge as there are no clear guidelines as to what constitutes sufficient knowledge. This was already performed in previous studies [19, 20, 23], but may, however, be debatable.

\section{Conclusions and implications}

To conclude, knowledge about benefits and harms of the MSP strongly differs by educational level and migration status. Especially women with a low educational level and immigrant women showed much lower chances for sufficient knowledge. Based on numerous critiques about the information brochure in Germany and requests that the information should be written in an easily understandable language [25], it has been revised in 2017 [26], thus, knowledge may have improved. It needs to be further evaluated, whether the new information brochure enables women, and especially women with low educational level and migration background, to have sufficient knowledge about benefits and harms of the MSP to enable them to make an informed choice for or against participation in the screening. A positive test result and a following diagnosis of breast cancer is oppressive and scary and might affect the mental and emotional health of women. Therefore, it is especially important that women know beforehand that a positive test result does not imply that it is breast cancer and that the possibility of an overdiagnosis is given. Furthermore, future research should explore standardized criteria of sufficient knowledge.

\section{Additional file}

Additional file 1: Table S1. Response frequencies for each knowledge item separated by educational level, migration status and received invitation. (DOCX $19 \mathrm{~kb}$ )

\section{Abbreviations}

Cl: Confidence interval; :. InEMa-study (Informierte Entscheidung zur Teilnahme am Mammographie-Screening-Programm); MSP: Mammography screening program; OR: Odds Ratio

Acknowledgements

We thank all women for their participation.
Authors' contributions

$E M B$, JS and PK conceived and coordinated the study. EMB and SK performed the analyses and drafted the manuscript. JS, PK, SVE and MR reviewed the manuscript and contributed to the interpretation of the results. All authors have read and approved the final manuscript.

\section{Funding}

This work was supported by the German Federal Ministry of Health within research for the National Cancer Plan, grant number NKP-332-028.

\section{Availability of data and materials}

The datasets generated and analysed during the current study are available from the corresponding author on reasonable request.

Ethics approval and consent to participate

The study was approved by the Ethics Committee of the Medical Faculty of Muenster University (2012-268-f-S) and the Data Protection Officer of Bielefeld University. Informed consent was obtained from all individual participants included in the study.

Consent for publication

Not applicable.

Competing interests

The authors declare that they have no competing interests.

\section{Author details}

'Department of Health Services Research and Nursing Science, Interdisciplinary Center for Health Literacy Research, School of Public Health, Bielefeld University, P.O. Box 1001 31, 33501 Bielefeld, Germany. ${ }^{2}$ Unit of Epidemiology and Biostatistics, Institute of Global Health, University Hospital Heidelberg, Heidelberg, Germany. ${ }^{3}$ Department of Public Health, Brandenburg University of Technology, Senftenberg, Germany. ${ }^{4}$ Department of Prevention and Health Promotion, School of Public Health, Bielefeld University, Bielefeld, Germany. ${ }^{5}$ Institute of Psychology, University of Hildesheim, Hildesheim, Germany.

Received: 16 April 2019 Accepted: 22 May 2019

Published online: 11 June 2019

\section{References}

1. Robert-Koch-Institut. Brustkrebs (Mammakarzinom). https://www.krebsdaten. de/Krebs/DE/Content/Krebsarten/Brustkrebs/brustkrebs_node.html (Accessed 23 May 2018)

2. Gemeinsamer Bundesausschuss. Richtlinie des Gemeinsamen Bundesausschusses über die Früherkennung von Krebserkrankungen (Krebsfrüherkennungs-Richtlinie / KFE-RL). https://www.g-ba.de/downloads/ 62-492-510/RL_KFU_2010-12-16.pdf (.

3. Kooperationsgemeinschaft Mammographie. Jahresbericht Evaluation 2015 Deutsches Mammographie-Screening-Programm. https://newsroom. mammo-programm.de/download/fachpublikation/KOOPMAMMO_ Jahresbericht_EVAL2015_20170926_web.pdf. Accessed 6 Nov 2018.

4. Helou A. Krebsfrüherkennung im Nationalen Krebsplan: Gesundheitspolitischer Rahmen und gesetzliche Neuregelungen. Bundesgesundheitsblatt Gesundheitsforschung Gesundheitsschutz. 2014; 57(3):288-93.

5. Gøtzsche PC, Nielsen M. Screening for breast cancer with mammography. Cochrane Database of Syst Rev. 2009:4:CD001877.

6. Marckmann G, In der Schmitten J. Krebsfrüherkennung aus Sicht der PublicHealth-Ethik. Bundesgesundheitsblatt Gesundheitsforschung Gesundheitsschutz. 2014;57(3):327-33.

7. Berens EM, Reder M, Razum O, et al. Informed choice in the German mammography screening program by education and migrant status: survey among first-time invitees. PLoS One. 2015;10(11):e0142316.

8. Gigerenzer G, Mata J, Frank R. Public knowledge of benefits of breast and prostate cancer screening in Europe. J Natl Cancer Inst. 2009;101(17):1216-20.

9. Albert US, Kalder M, Schulte $H$, et al. Das populationsbezogene Mammografie-Screening-Programm in Deutschland: Inanspruchnahme und erste Erfahrungen von Frauen in 10 Bundesländern. Gesundheitswesen. 2012;74(2):61-70. 
10. Webster P, Austoker J. Women's knowledge about breast cancer risk and their views of the purpose and implications of breast screening - a questionnaire survey. J Public Health. 2006;28(3):197-202.

11. Gummersbach E, in der Schmitten J, Mortsiefer A, et al. Willingness to participate in mammography screening: a randomized controlled questionnaire study of responses to two patient information leaflets with different factual content. Dtsch Arztebl Int. 2015;112(5):61.

12. Schulte H, Nass-Griegoleit I. Inanspruchnahme des qualitätsgesicherten Mammographie-Screenings - Follow Up Studie 2012. https://www. bundesgesundheitsministerium.de/fileadmin/Dateien/5_Publikationen/ Praevention/Berichte/Abschlussbericht_Mammographie-Screening_FollowUp_Studie_2012.pdf (Accessed 07 Nov 2014).

13. Dierks ML, Schmacke N. Mammografie-Screening und informierte Entscheidung - mehr Fragen als Antworten. Gesundheitsmonitor. 2014;1:1-16.

14. Domenighetti G, D'Avanzo B, Egger M, et al. Women's perception of the benefits of mammography screening: population-based survey in four countries. Int J Epidemiol. 2003;32(5):816-21.

15. van Agt $H$, Fracheboud J, van der Steen A, et al. Do women make an informed choice about participating in breast cancer screening? A survey among women invited for a first mammography screening examination. Patient Educ Couns. 2012;89(2):353-9.

16. Webster $P$, Austoker J. Does the English breast screening Programme's information leaflet improve women's knowledge about mammography screening? A before and after questionnaire survey. J Public Health. 2007; 29(2):173-7.

17. Schonberg MA, McCarthy EP, York M, et al. Factors influencing elderly women's mammography screening decisions: implications for counseling. BMC Geriatr. 2007;7(1):26.

18. Berens EM, Reder M, Kolip P, et al. A cross-sectional study on informed choice in the mammography screening programme in Germany (InEMa): a study protocol. BMJ Open. 2014;4(9):e006145.

19. Marteau TM, Dormandy E, Michie S. A measure of informed choice. Health Expect. 2001;4(2):99-108.

20. Mathieu E, Barratt AL, McGeechan K, et al. Helping women make choices about mammography screening: an online randomized trial of a decision aid for 40-year-old women. Patient Educ Couns. 2010;81(1):63-72.

21. Reder M, Kolip P. Does a decision aid improve informed choice in mammography screening? Results from a randomised controlled trial. PLoS One. 2017:12(12):e0189148

22. Reder M, Berens EM, Spallek J, Kolip P. Development of the Informed Choice in Mammography Screening Questionnaire (IMQ): Factor structure, reliability, and validity. BMC Psychology. 2019;7(17). https://doi.org/10.1186/ s40359-019-0291-2.

23. Smith SK, Barratt A, Trevena L, et al. A theoretical framework for measuring knowledge in screening decision aid trials. Patient Educ Couns. 2012;89(2):330-6.

24. Kickbusch I, Pelikan JM, Apfel F, et al. Health literacy: the solid facts. Copenhagen: World Health Organization; 2013

25. Gigerenzer $\mathrm{G}$, Wegwarth $\mathrm{O}$. Risikoabschätzung in der Medizin am Beispiel der Krebsfrüherkennung. Z Evid Fortbild Qual Gesundhwes. 2008;102(9):513-9.

26. Gemeinsamer Bundesausschuss. Mammographie-Screening: Eine Entscheidungshilfe. Programm zur Früherkennung von Brustkrebs für Frauen zwischen 50 und 69 Jahren. 2017.

\section{Publisher's Note}

Springer Nature remains neutral with regard to jurisdictional claims in published maps and institutional affiliations.

Ready to submit your research? Choose BMC and benefit from:
- fast, convenient online submission
- thorough peer review by experienced researchers in your field
- rapid publication on acceptance
- support for research data, including large and complex data types
- gold Open Access which fosters wider collaboration and increased citations
- maximum visibility for your research: over 100M website views per year
At BMC, research is always in progress.
Learn more biomedcentral.com/submissions

